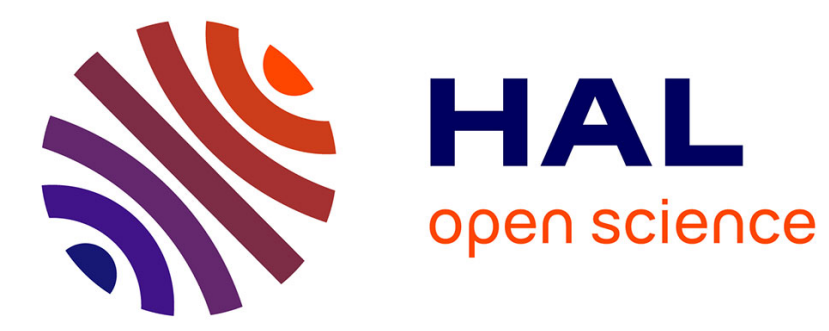

\title{
Energy Optimization in Redundant WSNs under Deterministic and Probabilistic Sensing Models
}

Ibrahima Diané, Rahim Kacimi, Zoubir Mammeri, Ibrahima Niang

\section{To cite this version:}

Ibrahima Diané, Rahim Kacimi, Zoubir Mammeri, Ibrahima Niang. Energy Optimization in Redundant WSNs under Deterministic and Probabilistic Sensing Models. IEEE 80th Vehicular Technology Conference (VTC 2014 - Fall), IEEE Vehicular Technology Society, Sep 2014, Vancouver, Canada. pp.1-5, 10.1109/VTCFall.2014.6965959 . hal-03252285

\section{HAL Id: hal-03252285 \\ https://hal.science/hal-03252285}

Submitted on 8 Jun 2021

HAL is a multi-disciplinary open access archive for the deposit and dissemination of scientific research documents, whether they are published or not. The documents may come from teaching and research institutions in France or abroad, or from public or private research centers.
L'archive ouverte pluridisciplinaire HAL, est destinée au dépôt et à la diffusion de documents scientifiques de niveau recherche, publiés ou non, émanant des établissements d'enseignement et de recherche français ou étrangers, des laboratoires publics ou privés. 


\title{
Energy Optimization in Redundant WSNs under Deterministic and Probabilistic Sensing Models
}

\author{
Ibrahima Diané ${ }^{1}$, Rahim Kacimi ${ }^{1}$, Zoubir Mammeri ${ }^{1}$, Ibrahima Niang ${ }^{2}$ \\ ${ }^{1}$ IRIT-UPS, University of Toulouse, France \\ ${ }^{2}$ LID-UCAD, University of Dakar, Senegal \\ \{diane, kacimi, mammeri\}@irit.fr, iniang@ucad.sn
}

\begin{abstract}
Almost all WSNs are deployed with some redundancy degree which is used only for robustness objectives. If not handled in an intelligent way, redundancy results in energy wasting because of redundant transmission and reception operations. In view of this energy wasting, a redundancy detection method under sensing models is proposed. We propose to take benefit from measurement redundancy to optimize the energy consumption and improve the end-to-end delay. We propose OER (Optimization of Energy based on Redundancy) protocol, to improve energy consumption in WSNs.

Index Terms-Wireless sensor networks, measurement redundancy, geographical proximity, sensing models
\end{abstract}

\section{INTRODUCTION}

WSNs deployment raises problems of power management and end-to-end communication delay. Indeed, despite the use of clustering schemes, WSNs are still faced with energy consumption and delay issues. Most of existing work propose improvements in terms of energy consumption. However, these solutions do not consider measurement redundancy and use data aggregation and sleep periods to improve energy consumption and delay.

When the network size becomes large, its management raises difficulties. The structuring of a sensor network is one of the main mechanisms to save energy in each node, which allows to extend the system lifetime. One of the best known structures is the hierarchy. The hierarchization technique enables to partition the network into subsets in order to facilitate its management. In such a technique, network view becomes local; special nodes may have additional roles. The literature includes several contributions on hierarchization techniques of sensor networks. This article advocates the use of geographical proximity and probabilistic sensing model for energy saving improvement. Indeed, we argue, in favor of a grouping technique where the nodes having redundant reading should be grouped. The measurement redundancy is the fact that several sensors in a same geographical proximity, report a same measurement value. Thus, the core idea of our approach is to determine and to group redundant nodes in order to have only one representing node by group which detect the eventual events and sends its data.
The rest of this paper is organized as follows. Related work in the field of hierarchical power management in WSNs is summarized in section II. Section III describes OER protocol and details the grouping technique based on the geographical proximity and the probability to detect events. In Section IV, we evaluate the performance of OER. Finally, we conclude this work in Section V.

\section{RElated WORK}

Low Energy Adaptive Clustering Hierarchy (LEACH) [6] is a cluster-based approach in which clusters are formed in a self-organized way. Each cluster is controlled by a cluster-head $(\mathrm{CH})$. With the aim of maximizing the network lifetime, the selection of the $\mathrm{CHs}$ is based on the residual energy level of nodes. CHs collect data from their cluster member nodes and, after processing, communicate the results to a global sink node. In order to reduce the amount of transmitted data to the sink, the $\mathrm{CHs}$ aggregate the collected data by their member nodes, and send an aggregated packet to the sink. However, although LEACH may increase the network lifetime, it has some limitations.

Unlike LEACH, where nodes self-configure themselves into clusters, LEACH-C [5] utilizes the sink for cluster formation. The sink receives information regarding the location and energy level of each node in the network. Using this information, the sink finds a predetermined number of $\mathrm{CHs}$ and organizes the network into clusters. The clusters are chosen to minimize the energy required for non- $\mathrm{CH}$ nodes to transmit their data to their respective CHs. The results presented in [5] indicate a definite improvement over LEACH.

In [3], the authors proposed a hierarchical protocol based on LEACH. The CHs formed in LEACH are grouped and organized in a hierarchy. They showed that the energy consumption decreases when the number of levels of the hierarchy increases. However, they did not improve the end-to-end delay of LEACH.

Hierarchical-PEGASIS [11], which is an extension to PEGASIS, is designed to address the delay incurred by packets during transmission to the base station. In order to improve the performance by reducing the delay in PEGASIS, messages are transmitted simultaneously. Another similar protocol to PEGASIS called $C^{2} E^{2} S$, has been proposed in [8]. It is based on clusters and chains. It 
is a centralized protocol where the base station organizes the network on the basis of energy information of nodes.

TEEN protocol [12] is another hierarchical protocol coupled with data centric approach that is designed to react to sudden changes in the sensing attributes such as weather conditions. TEEN approach is more proactive rather than reactive. This is particularly important for time-sensitive applications. The sensor network architecture is based on a hierarchical grouping where closer nodes form clusters and this process goes on the second level until base station (sink) is reached. However, TEEN is not suitable for applications where periodic data are required since the user may not get any data at all if the thresholds are not reached.

APTEEN proposed in [13] is an extension to TEEN and designed to capture both periodic data and reacting to time-critical events. The architecture is similar to TEEN. The main drawbacks are the overhead and complexity of forming clusters in multiple levels, implementing threshold-based functions and dealing with attributebased naming of queries.

In [2], [1], the authors proposed a clustering approach called Virtual Grid Architecture (VGA) to maximize the lifetime of WSNs where nodes are stationary or move at low speed. They used GPS-free approach [14] to build clusters. In VGA, the area of deployment of sensors is formed in a rectilinear virtual topology containing of small areas in the shape of a square, and in each of them, a node is selected as $\mathrm{CH}$. The data aggregation is performed at two levels: local and global. The local aggregation is performed by all CHs also called Local Aggregators (LAs), while the global aggregation is performed by a subset of LAs, called Master Aggregators (MAs). However, the determination of all MAs is a hard problem.

The authors in [9], [10] proposed a self-organisation technique based on the clustering approach to optimize the energy consumption in sensor networks. This technique consists in grouping the nodes close geographically in clusters. It requires determining parameters to produce a small number of clusters, homogeneous clusters (in size and radius), and stable clusters. Two thresholds are used to control the size of generated clusters and the distances between cluster members and their corresponding clusterhead are at most two hops. However, no solutions were provided for thresholds settings and inter-cluster communication.

We have proposed MR-LEACH [4], which is an extension to the wellknown LEACH protocol to improve energy consumption in cluster-based WSNs. MR-LEACH combines geographical proximity of the nodes and their readings redundancy in order to reduce the number of the intracluster transmissions. In the first stage, the $\mathrm{CHs}$ collect and observe the cluster readings, while in the second they determine the measurement redundancy and group the redundant nodes. After identifying the redundancy groups, only one representing node will send its readings and the other nodes stop their transmissions. However, MR-LEACH uses memory space to collect data to analyze.

\section{REDUNDANCY IN WIRELESS SENSOR NETWORKS}

\section{A. Problem statement}

WSNs are still faced with problems of energy consumption and delay. The initial node placement may explicitly specify some redundancy in the deployed sensor nodes. The measurement redundancy may result from this high density of nodes. Multiple neighboring nodes, which are placed at specific locations and/or at given distance each from other, may detect the same event and/or report the same value. It is therefore necessary to propose mechanisms to manage possible measurement redundancy in order to improce the network lifetime.

This article proposes and analyzes OER (Optimization of Energy based on Redundancy), a technique which reduces the measurement redundancy in WSNs.

\section{B. Partitioning technique by geographical proximity}

OER uses a partitioning technique based on geographic proximity. Depending on the geographic location, our technique allows the grouping of the nodes which detect the same events. Thus, only the representing nodes send their packets. This way, the communications are reduced and the energy consumption of the nodes is optimized.

1) Assumptions: For easier understanding of our scheme in the remainder of this paper, we make some reasonable assumptions.

- Nodes are densely and randomly deployed on a square area and their placement is known.

- Nodes observe the physical events, generate a monitoring traffic and send it to the BS.

2) Principe of our approach: OER combines geographical proximity of the nodes and their probability to detect the same events in order to reduce the number of the transmissions. In the first stage, we determine and group the redundant nodes, while in the second we determine if the partitioning is best minimizing an objective function. The process is repeated until an optimal partitioning is found. We give more details hereafter.

- As soon as the sensor nodes are deployed on the network, the starting of uncertainty in sensor detection is used to form the groups of redundant nodes. The value of objective function is also determined.

- After the redundant groups set-up, using the value of objective function, we verify if the new partitioning is better. So we designate a function that serves as a criterion to determine the best solution to our partitioning problem. The goal of our optimization problem is then to minimize this function by using a tabu search method.

After the initialization phase, OER enters in the sensing and transmission phase. Thus, only one representing node will be active at a time in a same redundancy group. The 
representing nodes detect a possible event and send their data to the base station.

Geographical proximity grouping method: The geographical proximity exists if the distance between the nodes is less than the given threshold $d$ and that these nodes detect the same events. This threshold is deduced directly from the starting of uncertainty in sensor detection.

Let the Euclidean distance between a sensor $s_{i}$ and a point $p$ in the given region be $d_{2}\left(s_{i}, p\right)$. In the binary disk model, the maximum sensing range of the node $s$ is parameterized by $R_{\max }$. The target at point $p$ can be detected by the sensor $s$ if and only if $d_{2}(s, p) \leq R_{\text {max }}$. The probability that an event at a distance $d(s, p)$ from the node will be detected is:

$$
p_{\text {det }}(x)= \begin{cases}1, & x \leq R \\ 0, & \text { otherwise }\end{cases}
$$

where, $x=d_{2}(s, p)$. If $p_{\text {det }}(x)=1$, it means that the target at point $p$ can be detected by sensor $s$.

According to the Elfes sensing model [7], the probability that a sensor detects an event to a distance $x$ is:

$$
p_{\text {det }}(x)= \begin{cases}1, & x \leq R \\ e^{-\lambda(x-R)^{\beta}}, & R<x \leq R_{\max } \\ 0, & \text { otherwise }\end{cases}
$$

where, $R$ defines the starting of uncertainty in sensor detection and the parameters $\lambda$ and $\beta$ are adjusted according to the sensor physical properties. $R_{\max }$ is the maximum sensing range of the node.

To partition the network, we group the redundant nodes according to the selected nodes as centers of the event regions. For this, we start from a randomly selected node that serves as center of a first region of events. We group redundant nodes of this region. Thereafter we move the center to another node to form the next event region and group the corresponding redundant nodes. This process is repeated until each node is member of one group. For that there is geographical proximity between a set of nodes and a redundancy, it is necessary that:

$$
d_{2}\left(N_{i}, C_{c}\right) \leq R-R_{e v}
$$

and therefore,

$$
d_{2}\left(N_{i}, N_{j}\right) \leq 2\left(R-R_{e v}\right)
$$

where, $C_{c}$ defines the center of the current event region, $R_{e v}$ defines the radius of events regions and the $i^{t h}$ node is like a point $N_{i}$ in the space that can be represented as $N_{i}=\left(x_{i}, y_{i}\right)$. Under the probabilistic sensing model, we consider a threshold probability $\left(P_{t}\right)$ to determine the redundant nodes. Equation (3) can be approximated for $R<x \leq R_{\max }$ and $\beta=1$ as

$$
p_{\text {det }}(x)=e^{-\lambda(x-R)}
$$

Considering the threshold probability $\left(P_{t}\right)$, we have:

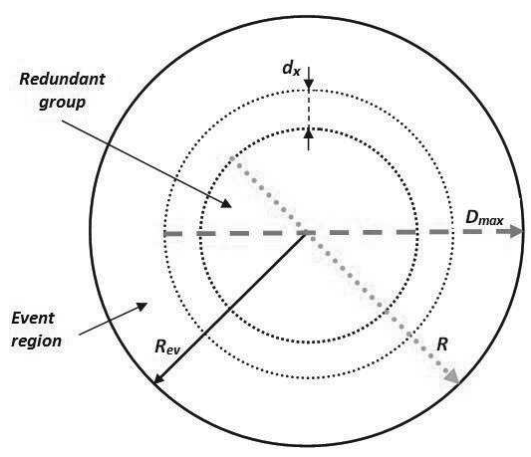

Fig. 1. Sensing for redundancy nodes

$$
P_{t}=e^{-\lambda\left(D_{\max }-R\right)}
$$

and therefore,

$$
D_{\max }=R-\frac{\ln \left(P_{t}\right)}{\lambda}
$$

where, $P_{t}$ defines the probability that a sensor detects an event to a distance $D_{\max }$. For that there is redundancy between a set of nodes at a probability $P_{t}$, it is necessary that:

$$
\begin{gathered}
d_{2}\left(N_{i}, N_{j}\right) \leq 2\left(D_{\max }-R_{e v}\right) \\
d_{2}\left(N_{i}, N_{j}\right) \leq 2\left(R-\left(\frac{\ln \left(P_{t}\right)}{\lambda}+R_{e v}\right)\right)
\end{gathered}
$$

The nodes are densely and randomly deployed on a square area and their placement is known. BS creates a table of coordinates where it will store the reported coordinates by the sensor nodes. To form a redundant group, we first consider an unvisited node (random sampling without replacement). Then we determine all redundant nodes of the event region whose center is the previously selected node. A redundant group is thus formed. Finally, all nodes in the formed group are marked as visited as detailed in Algorithm 1.

Objective function: We propose a function that serves as a criterion to determine the optimal solution to our partitioning problem. Specifically, it associates a value with each instance of our problem. Let a network of $N$ deployed sensors in an area. The algorithm 1 seeks to group redundant nodes and thus create the partitions of nodes. This is an algorithm receiving as input the set of nodes and provides $k$ partitions as output.

By considering a partitioning $P$ with $k$ partitions, $P=\left\{p_{1}, p_{2}, \ldots, p_{k}\right\}(k \leq N)$. A packet transmission is performed by one node of the partition 1 or by one node of the partition 2 or ... by one node of the partition $k$. The average energy consumption of a node is written by:

$$
E(P)=\left(\frac{1}{k} \cdot \frac{1}{\left|p_{1}\right|}+\frac{1}{k} \cdot \frac{1}{\left|p_{2}\right|}+\cdots+\frac{1}{k} \cdot \frac{1}{\left|p_{k-1}\right|}+\frac{1}{k} \cdot \frac{1}{\left|p_{k}\right|}\right) e_{t_{x}}
$$




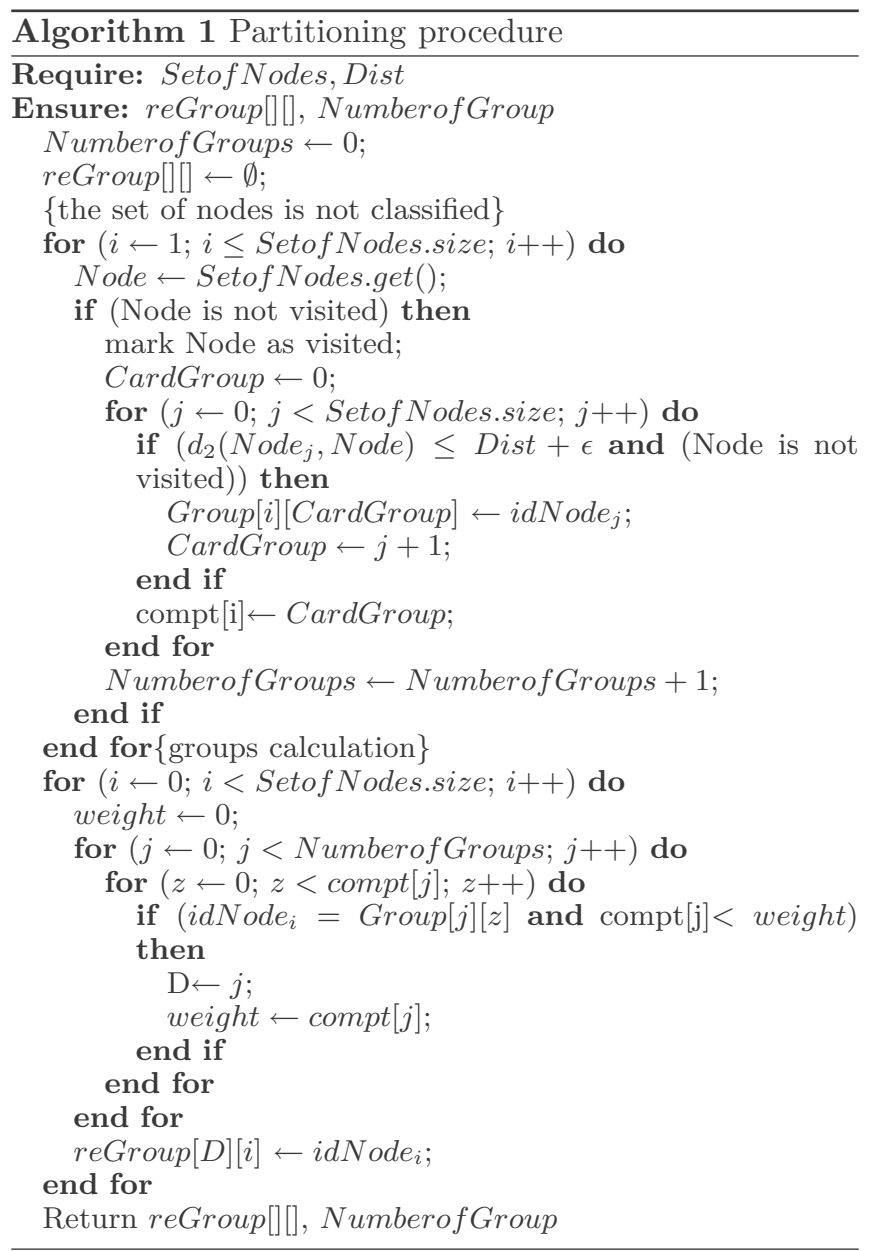

$$
E(P)=\frac{1}{k} \sum_{i=1}^{k} \frac{1}{\left|p_{i}\right|} e_{t_{x}}
$$

where $k$ is number of partitions, $\left|p_{i}\right|$ is the cardinal of the partition $i, e_{t_{x}}$ the energy consumed by transmission of one packet, and $E(P)$ is the average energy consumed by a node under the partitioning $P$. The average energy consumed during a superframe, with partitioning $P$, is written by:

$$
E_{G}(P)=\sum_{i=1}^{k} \frac{1}{\left|p_{i}\right|} e_{t_{x}}
$$

After designing our objective function, we do a tabu search to find an optimal solution (a partitioning with the lowest possible energy consumption) as detailed in Algorithm 2. After having found an optimal partitioning, only the representing nodes have to send data to the BS.

\section{Performance Evaluation}

To evaluate the performance of OER, we used NS2 simulation tool which provides an opportunity to integrate the news protocols.

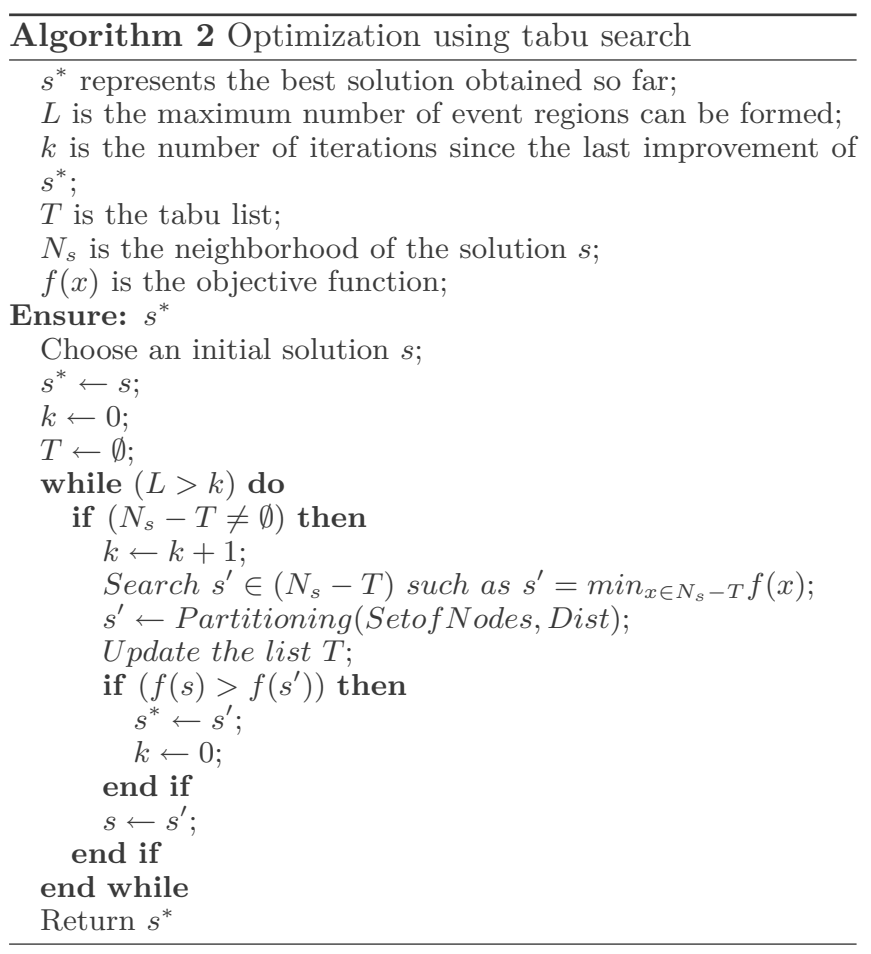

\section{A. Simulation set-up}

We used a network scenario consisting of 100 to 1000 nodes randomly deployed over a $250 * 250 \mathrm{~m}^{2}$ area. The sink's coordinates are $(135,155)$. The simulation parameters are as follows:

TABLE I

SimUlation PARAMETERS

\begin{tabular}{|r|c|}
\hline Packet length & 55 bytes \\
\hline Initial node energy & $2 \mathrm{j}$ \\
\hline Transmission bit rate & $250 \mathrm{kbs}^{-1}$ \\
\hline Transmit power & $24.75 \mathrm{~mW}$ \\
\hline Receive power & $13.5 \mathrm{~mW}$ \\
\hline Idle power & $6.5 \mathrm{~mW}$ \\
\hline Sleep power & $15 \mu \mathrm{W}$ \\
\hline Starting of uncertainty in sensor detection $R$ & $50 \mathrm{~m}$ \\
\hline Radius of event region $R_{e v}$ & $25 \mathrm{~m}$ \\
\hline
\end{tabular}

\section{B. Simulation results}

From Fig. 2, we can see that when the network size increases the energy consumption of the nodes in OER decreases. Indeed, with high density the measurement redundancy increases. Thus, the sink receive less data. This is because, the number of redundant groups decreases which reduces the number of transmissions in the network. So, when the number of nodes is being higher, they are grouped and only the group representing nodes send their packets in the network. Furthermore, the curves of Fig. 2 also show that the performance gap between boolean sensing model and probabilistic sensing model becomes more significant when the event detection probability decreases. 


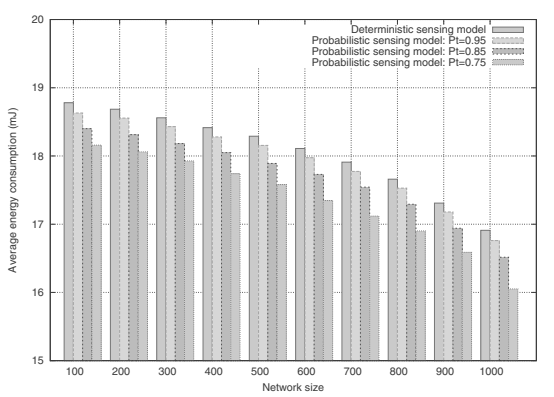

Fig. 2. Average energy consumption of each node during one round

Fig. 3 shows how the network lifetime is affected over time

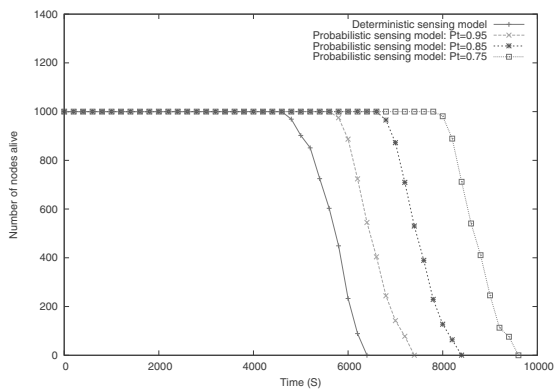

Fig. 3. Network lifetime

using boolean sensing model versus probabilistic sensing model. In this set of experiments, we consider a network size of 1000 sensor nodes. Probabilistic sensing model has a greater network lifetime compared with boolean sensing model. OER under probabilistic sensing model, is more effective to extending the network lifetime. Compared to OER under boolean sensing model, it takes approximately 1.7 times longer until the first node dies. Fig. 4 plots

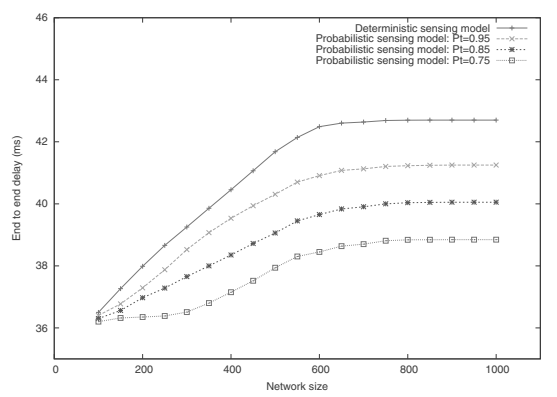

Fig. 4. End-to-end delay

the results of the average end-to-end delay evolution. As first result, we can see that the average delay grows with increasing of the network size. However, this increasing is less significant for OER under probabilistic sensing model, due to the reduced number of redundant groups. In fact, the sink in OER under probabilistic sensing model, expects a number of packets $k \ll n$, where $n$ is the size of the cluster.

\section{Conclusion}

This paper addresses energy efficiency in WSNs. Our solution consists in taking benefit from the measurement redundancy. We proposed OER protocol, to improve energy consumption in WSNs. OER has several strengths. It combines the sensing models and the geographical proximity of the nodes with the measurement redundancy in order to improve the energy efficiency and provides better endto-end communication delays. Furthermore, we assessed how it outperforms the protocols based on deterministic sensing model. In our future work, we would like to extend our approach to consider initial WSN deployment including explicit redundancy specification.

\section{REFERENCES}

[1] J.N. Al-Karaki, R. Ul-Mustafa, and A.E. Kamal. Data aggregation in wireless sensor networks - exact and approximate algorithms. In Workshop on High Performance Switching and Routing, 2004.

[2] J.N. Al-Karaki, R. Ul-Mustafa, and A.E. Kamal. Data aggregation and routing in wireless sensor networks: Optimal and heuristic algorithms. Computer Networks, 53(7):945 - 960, 2009.

[3] S. Bandyopadhyay and E.J. Coyle. An energy efficient hierarchical clustering algorithm for wireless sensor networks. In Proc. of 22 Annual Joint Conference of the IEEE Computer and Communications, INFOCOM'03, 2003.

[4] Ibrahima Diané, Rahim Kacimi, Zoubir Mammeri, and Ibrahima Niang. Energy optimization based on the redundancy in wsns. In 6th Joint IEEE IFIP Wireless and Mobile Networking Conference. IEEE, 2013.

[5] W.B. Heinzelman, A.P. Chandrakasan, and H. Balakrishnan. An application-specific protocol architecture for wireless microsensor networks. Wireless Communications, IEEE Transactions on, 1(4):660-670, October 2002.

[6] W.R. Heinzelman, A. Chandrakasan, and H. Balakrishnan. Energy-efficient communication protocol for wireless microsensor networks. In Proc. of the 33rd Annual Hawaii International Conference on System Sciences, 2000.

[7] Ashraf Hossain, PK Biswas, and S Chakrabarti. Sensing models and its impact on network coverage in wireless sensor network. In IEEE Region 10 and the Third international Conference on Industrial and Information Systems. IEEE, 2008.

[8] T.T. Huynh and C.S. Hong. An energy* delay efficient multihop routing scheme for wireless sensor networks. IEICE transactions on information and systems, 89(5):1654-1661, 2006.

[9] M. Lehsaini, H. Guyennet, and M. Feham. A novel clusterbased self-organization algorithm for wireless sensor networks. In International Symposium on Collaborative Technologies and Systems, 2008.

[10] M. Lehsaini, H. Guyennet, and M. Feham. An efficient clusterbased self-organisation algorithm for wireless sensor networks. International Journal of Sensor Networks, volume 7:85-94, March. 2010 .

[11] S. Lindsey, C. S. Raghavendra, and M. Sivalingam, Krishna. Data gathering in sensor networks using the energy delay metric. In Proc. of the 15th International Parallel $\&$ Distributed Processing Symposium, IPDPS'01, Washington, DC, USA, 2001.

[12] A. Manjeshwar and D.P. Agrawal. Teen: a routing protocol for enhanced efficiency in wireless sensor networks. In Proc. of International Parallel and Distributed Processing Symposium, pages $2009-2015$, april 2001.

[13] A. Manjeshwar and D.P. Agrawal. Apteen: a hybrid protocol for efficient routing and comprehensive information retrieval in wireless sensor networks. In Proc. of International Parallel and Distributed Processing Symposium, IPDPS'02, 2002.

[14] A. Savvides, C.C. Han, and M.B. Strivastava. Dynamic finegrained localization in ad-hoc networks of sensors. In International Conference on Mobile Computing and Networking, volume 2001, pages 166-179, 2001. 